\title{
Características Psicométricas del Cuestionario de Abuso en Contra de la Mujer, Versión en Español: Su Uso en Investigación y Práctica Clínica
}

\section{Psycometric Characteristic of the Women Abuse Screen, Spanish Versión: It Uses in Research and Practice}

\author{
Ana M. Linares \\ University of Kentucky, Lexington, Kentucky, USA \\ Universidad de Tarapacá, Arica, Chile. \\ Jane Dimmitt Champion \\ University of Texas at Austin, School of Nursing Austin, Texas, USA \\ Bertha C. Salazar \\ Universidad Autónoma de Nuevo León, Monterrey, México.
}

(Rec: 3 de mayo de 2012 / Acept: 9 de julio de 2013)

\begin{abstract}
Resumen
Este artículo describe el proceso de traducción y adaptación del inglés-español del instrumento "Woman Abuse Screen" para uso en investigación. El cuestionario original fue traducido al español por dos traductores e intérpretes bilingües, y posteriormente se condujo una revisión de contenido por expertos asegurándose que incluía elementos relevantes del concepto "violencia". El instrumento traducido "Cuestionario de Abuso en contra de la Mujer" (CACM) fue puesto a prueba con 217 mujeres que iniciaron su control prenatal en clínicas en Monterrey, México. El análisis factorial exploratorio con rotación Varimax agrupó nueve reactivos en tres factores (violencia sexual, física y psicológica) con varianza explicada de $79.87 \%$. El coeficiente de confiabilidad obtenido fue 0.86 (Alfa de Cronbach). Se concluye que CACM es un instrumento fácil de contestar y que entrega información sobre el tipo de violencia que está siendo víctima la mujer en el presente o el pasado. CACM puede ser utilizado en investigación y/o práctica clínica.

Palabras clave: violencia, instrumento, validación, embarazo, México.
\end{abstract}

\begin{abstract}
This paper describes the process of English-Spanish translation and adaptation of the "Woman Abuse Screen" for use in research. The original screen was initially translated into Spanish by two bilingual translatorsinterpreters, followed by a content review conducted by experts to ensure that the screen included relevant aspects of the conceptualization of violence. The Spanish translation "Cuestionario de Abuso en contra de la Mujer" (CACM) was tested with 217 pregnant women initiating prenatal care in outpatient clinics in Monterrey, Mexico. Exploratory factor analysis with Varimax rotation grouped the nine items on three factors (sexual, physical and psychological violence) with an explained variance of $79.87 \%$. A reliability coefficient of 0.86 was obtained (Cronbach Alpha). It is concluded that CACM is easy to answer and provides information on previous and present violence experienced by the women. CACM may be utilized in practice and research for screening of interpersonal violence.

Key words: Violence, Instrument, Validation, Pregnancy, Mexico.
\end{abstract}

Correspondencia: to Ana M. Linares, Assistant Professor, College of Nursing, 315 CON Building, University of Kentucky, Lexington, KY 40536.

USA. 1-859-323-4726. Emails: a.quelopana@uky.edu aquelopa@uta.cl 


\section{Introducción}

La violencia en contra de la mujer embarazada se considera un problema de salud pública preponderante. Investigadores de diferentes disciplinas han estudiado esta problemática a nivel mundial tanto desde una perspectiva cualitativa como cuantitativa. En la actualidad, hay evidencia científica que reconoce múltiples riesgos de morbilidad y mortalidad asociados a violencia en contra de la mujer embarazada, entre ellos tenemos: problemas durante el embarazo, parto prematuro, recién nacido de bajo peso de nacimiento, mayor probabilidad de infecciones de transmisión sexual, uso de sustancias nocivas como cigarrillo, alcohol y drogas, depresión y desordenes post-traumático (El-Mohandes, Kiely, Gantz y El-Khorazaty, 2011; Humphreys, 2011; Shah y Shah, 2010; Stampfel, Chapman y Álvarez, 2010). Además, violencia durante el embarazo se ha asociado a embarazo no deseado e inadecuado control prenatal (CPN) (Miller et al., 2010; Moraes, Arana y Reichenheim, 2010; Quelopana, Champion y Salazar, 2007).

Es reconocido que el embarazo no es protector de violencia; algunos autores refieren que la violencia incrementa durante el embarazo (Castro, 2004; Castro, Peek-Asa y Ruiz, 2009; Flury, Nyberg y Riecher-Rossler, 2010; Jasinski, 2004) y puede llevar a homicidio (Chang, Berg, Salzman y Herndon, 2005; Cheng y Horon, 2010). Opuestamente algunos estudios reportan que el embarazo podría proveer una protección en contra de la violencia (Chu, Goowin y D'Angelo, 2010; Taylor y Nabors, 2009). Investigadores a nivel internacional reconocen que el embarazo es un tiempo muy beneficioso para consultar y pesquisar violencia en forma rutinaria, como una forma de prevenir riesgos de complicaciones (Bunn, Higa, Parker y Kaneshiro, 2009; Humphreys, Tsoh, Kohn y Gerbert, 2011; Onifade, Von-Rege, Mumford y Bewley, 2010; O’Reilly, Beale y Gillies, 2010; Shay-Zapien y Bullock, 2010). En México la Norma Oficial del Cuidado Médico de la Violencia Familiar (Secretaria General de Salud de México, 2000) recomienda el escrutinio rutinario de toda embarazada, sin embargo, se sabe que por ser un tema delicado muchos proveedores de salud prefieren ignorar esta recomendación (Bunn et al., 2009; Lazenbatt, Taylor y Cree, 2009). Valdez-Santiago, Arenas-Montreal y Hernández-Tezoquipa (2004) reportaron en un estudio que parteras Mexicanas reprodujeron una de las más frecuentes respuestas sociales frente a la violencia en contra de la mujer, esto es, el negar que ocurra distanciándose de la mujer como una forma de no verse involucradas. Revisiones de artículos relacionados con violencia reportan que las mujeres que han sido víctimas de violencia encuentran difícil compartir su experiencia o buscar ayuda con la policía o algún profesional de salud (Flury et al., 2010). Esto reafirma que muchas mujeres prefieren esconder los incidentes de violencia como una forma de mantener el problema dentro de los ámbitos del hogar y en donde la propia víctima, puede entrar en complicidad de los abusos creyendo que hay que aplacar, silenciar, esconder, minimizar, para no molestar a los actores protagónicos ni a sí mismo (Castro, 2004; Jacobson y Gottman, 2001; Ravazzola, 1997).

Para realizar pesquisa rutinaria de violencia se requiere que el personal de salud cuente con instrumentos validados de fácil aplicación y que puedan entregar información confiable. El objetivo principal de este artículo es reportar el proceso de traducción al Español y adaptación del instrumento "Woman Abuse Screen" (Champion, Piper, Holden, Korte y Shain, 2004) a la población Mexicana. Como segundo objetivo es mostrar los hallazgos encontrados en relación a violencia en el embarazo en una población de mujeres del área Metropolitana de Monterrey, México.

\section{Woman Abuse Screen}

Este instrumento originalmente fue diseñado para pesquisar la experiencia de violencia doméstica de mujeres residentes de los EE.UU. Para su diseño, las autoras utilizaron un enfoque cualitativo y además, requirió una cuidadosa revisión de literatura y reexaminación de instrumentos usados en estudios previos que midieron violencia doméstica (Champion, 1999; Champion, 1998; Champion, Shain, Piper y Perdue, 2001). El instrumento posee diez reactivos que indagan la historia de violencia de tipo psicológica, física y sexual en la mujer con su pareja actual y/o con alguna otra persona, ya sea con anterioridad o en el presente. La autora principal refiere que el instrumento se utiliza para pesquisar experiencias con violencia y no para medir la severidad de violencia. Es decir, un puntaje mayor se debe interpretar como mayor experiencia o historia de violencia y no precisamente una mayor severidad de esta. La definición de violencia que guió el desarrollo de este instrumento se ajustó a la dada por la Organización Mundial de la Salud (OMS, 1996) que la define como:

"Cualquier acto de violencia basado en género que dé como resultado o tenga la posibilidad de terminar en daño físico, mental o sexual hacia la mujer, incluyendo amenaza a la acción, coerción o arbitraria privación de la libertad, y que ocurra en público o en forma privada" (p. 1).

Este instrumento fue elegido, porque se ajusta a nuestra visión de violencia. Los instrumentos que miden severidad, 
según nuestra opinión, pueden caer en la subjetividad; simplemente porque en dichos instrumentos se considera que violencia física es más severa que la psicológica. Sin embargo, la literatura advierte que la violencia psicológica puede tener resultados tan o más devastadores que la violencia física (Pico-Alfonso et al., 2006).

Este instrumento ha sido utilizado múltiples veces en estudios de investigación en población de Estados Unidos, reportando consistencia interna (Alfa de Cronbach) entre 0.78 y 0.82 (Champion et al., 2004; Champion, 2005; Thurman et al., 2008).

\section{Método}

\section{Participantes}

Este estudio es un análisis secundario de datos obtenidos previamente (Quelopana, Champion y Salazar, 2009). Para su realización se obtuvo autorización de la Oficina de Investigación de la Universidad de Kentucky, previo visado por la Oficina de Investigación de la Universidad Autónoma de Nuevo León, Monterrey México, lugar donde originalmente se realizó la investigación. La muestra $(\mathrm{n}=217)$ corresponde a datos obtenidos con los siguientes criterios de inclusión: mujeres embarazadas, 18 años o más, y que iniciaron su CPN en clínicas del área Metropolitana de Monterrey, México. Las mujeres elegibles fueron llamadas a un cubículo privado asignado para la realización de la entrevista. Las embarazadas que aceptaron ser entrevistadas se les leyó el consentimiento informado y se les pidió que lo firmaran. Se realizó una entrevista estructurada para obtener información relacionada a características socio-demográficas, historia reproductiva, embarazo actual, percepción de apoyo social e historia de violencia. Todas las entrevistas fueron realizadas por la investigadora principal. El tiempo promedio de realización de la entrevista fue de $29(D E \pm 6.3)$ minutos por participante.

Para asegurar un manejo adecuado de las embarazadas que manifestaron historia de violencia, a cada una de las participantes se les informó las opciones a la que podía recurrir, se entregó asesoría y consejería y finalmente se ofreció si voluntariamente deseaba informarlo a la trabajadora social de la institución, quien pudo referirla a asesoría de tipo legal y/o psicológica.

\section{Instrumentos}

El Woman Abuse Screen (Champion et al., 2004) fue proporcionado por su autor principal, quien participó en el estudio. Para iniciar la adaptación de este cuestionario fue necesaria la traducción al español. Para esto, se entregó la versión en inglés a dos traductores-intérpretes bilingües; ambos profesionales contaban con reconocida experiencia en trabajos de traducción en ciencias de la salud. Posteriormente se realizó la traducción inversa (back translation) por lo que un segundo grupo de traductores que no conocían la versión en inglés tradujo la versión traducida al idioma original. Las versiones originales y traducidas se compararon con el fin de validar la exactitud de estas y descarta errores de traducción y/o la falta de texto y otros errores. Terminada el proceso de traducción, se realizó una revisión de contenido del instrumento por expertos. Para esto, un grupo de enfermeras expertas en cuidado prenatal más las investigadoras realizaron una revisión minuciosa para analizar si el cuestionario incluía los elementos relevantes del concepto de violencia que se pretendía medir en el estudio. El instrumento en español se le llamó: Cuestionario de Abuso en Contra de la Mujer (CACM). Este instrumento mantuvo nueve reactivos de los diez que contiene el instrumento original. La pregunta "alguna vez alguien ha tenido sexo con usted sin protección cuando usted quería usar protección en contra de Infecciones de Transmisión Sexual? fue excluida por sugerencia de los expertos revisores de la traducción; explicando que tal conducta tiene un componente cultural y no es considerado abuso en la población Mexicana. Con los nueve reactivos restantes se realizó un estudio piloto que reveló que el diseño y la frecuencia de las preguntas fueron de fácil comprensión para las participantes y que proporcionaban datos suficientemente variados (Quelopana et al., 2007). El instrumento final quedó con nueve reactivos que indagan la historia de violencia de tipo psicológica, física y sexual en la mujer con su pareja actual y con alguna otra persona, ya sea con anterioridad o en el presente (Apéndice A). Las respuestas son de tipo dicotómicas con sí $=1$ o no $=0$, y su puntaje oscila entre cero y nueve puntos. A mayor puntaje mayor historia de violencia.

\section{Análisis estadístico}

Con el fin de obtener reducción de los datos e identificar los grupos o conglomerados de variables relacionadas en el cuestionario, se realizó análisis factorial exploratorio (Burns y Grove, 2001). El análisis factorial exploratorio tiene como objetivo principal reducir un conjunto amplio de variables a un grupo de medidas más fácil de manipular; esto se logra esclareciendo las complejas interrelaciones entre variables e identificando cuales se enlazan como conceptos unificados (Polit y Hungler, 2000). 
$\mathrm{El}$ análisis factorial consta de dos fases independientes. La primera fase es la fase de extracción de factores y tiene como objetivo buscar agrupamientos de las variables que muestren una alta interrelación en la matriz de correlación; la segunda fase es la rotación de factores. El método de extracción que se usó en este estudio fue el método de componentes principales (factores principales o ejes principales). Con el resultado se obtuvo una matriz de factores sin rotar usándose como punto de corte para la extracción de factores Valores Característicos (eigenvalue) mayores que 1.00. La segunda fase correspondió a la rotación de factores. El objetivo de los ejes sin rotar es justificar un máximo de varianza, pero rara vez proporcionan una estructura con significado conceptual. Sin embargo, si se rotan de manera que los agrupamientos de variables se asocien claramente con un factor, las posibilidades de interpretación se incrementan (Polit y Hungler, 2000). En este caso se realizó rotación Varimax con normalización Kaiser; para la asignación de variables a un factor se usó supresión de valores absolutos inferiores a 0.40 .

Para medir homogeneidad o congruencia interna de la escala se obtuvo el coeficiente de confiabilidad Alfa de Cronbach. El Alfa de Cronbach nos ayuda a analizar si en condiciones ideales el conjunto de reactivos de la escala diseñada para medir un atributo, mide consistentemente el atributo crítico que dice medir (Burns y Grove, 2001; Polit y Hungler, 2000).

Se utilizó estadística descriptiva e inferencial obteniéndose frecuencias, proporciones, medidas de tendencia central y medidas de variabilidad de las variables socio-demográficas y de las características reproductivas de las participantes. Se analizó a través de Chi cuadrado las variables dicotómicas asociadas significativamente a historia de violencia. Se realizó correlación de Spearman para observar asociación de las variables independientes con violencia.

\section{Resultados}

\section{Validación del Cuestionario de Abuso en Contra de la Mujer (CACM)}

Este cuestionario contiene nueve reactivos que indagan sobre historia de violencia de tipo psicológica, física y sexual en el presente y en el pasado. El análisis de coeficiente de confiabilidad obtuvo un Alfa estandarizada 0.86. El análisis de factores reveló tres componentes en la matriz con una varianza explicada de $79.87 \%$. El primer factor explicó $51.03 \%$ de la varianza, el segundo factor $16.52 \%$ y el tercer factor $12.31 \%$. Se observa en la Tabla 1 que el primer componente agrupa tres reactivos que corresponden a violencia sexual, el segundo posee tres reactivos que corresponde a violencia psicológica y el tercer componente con tres reactivos de violencia física. El reactivo ¿alguien ha tratado de forzarla a hacer algo que no quería hacer, amenazando con lastimarla? cargó tanto en el componente uno como en el dos, pero se dejó en el dos por relacionarse más con este componente; lo mismo ocurre con el reactivo ¿alguien la ha puesto al suelo, dado puñetazos, pateado o tratado de estrangularla? que cargó en el componente dos y tres, pero se deja en el tres por relacionarse más con este componente.

\section{Características socio-demográficas de las participantes}

La edad promedio de las participantes fue de 25.5 ( $D E$ 5.9) años, con rango entre 18 a 46 años. El 80\% $(n=173)$ reportó vivir con pareja; el 55\% de estas mujeres reportó estar casada y el resto vivía en unión libre. En relación al nivel educacional, el 75\% $(n=162)$ tenía escuela secundaria terminada. El 42\% del grupo reportó ingresos económicos de $<\$ 4000$ pesos mexicanos por mes (aproximadamente 400 dólares); $\geq \$ 4000$ mexicanos por mes fue igualmente reportado por el $42 \%$ del grupo; y $16 \%$ no supo la información. Sólo el $25 \%$ del grupo de participantes refirió trabajar fuera de casa. Finalmente, el $73 \%$ reportó que el número de personas que vivían en el hogar fue de 5 o menos personas.

\section{Embarazo actual e historia reproductiva}

Sólo el 33\% de las participantes estaban cursando su primer embarazo. El 54\% de las embarazadas inició el CPN durante el primer trimestre del embarazo; el 34\% lo inició en el segundo trimestre; y el $12 \%$ en el tercer trimestre. De las participantes multíparas (mujeres con embarazos previos) el 70\% refirió haber tenido algún problema en embarazos anteriores tales como infección del tracto urinario, anemia, hipertensión del embarazo, síntoma de parto prematuro, entre otros.

\section{Historia de violencia}

El número de mujeres que reportaron historia de violencia correspondió al 34.6\% $(\mathrm{n}=75)$, de las cuales, el $84 \%$ $(n=63)$ reportó violencia psicológica, el 64\% $(n=48)$ violencia física y el $19 \%(\mathrm{n}=14)$ violencia de tipo sexual. Las respuestas individuales a los reactivos de violencia psicológica, física y sexual fueron: ¿alguna vez: a) alguien la ha criticado o reprimido? $(23 \%)$; b) ¿le ha dado miedo 
expresar lo que piensa por que se enojan con usted? (19\%); c) ¿alguien ha tratado de forzarla a hacer algo que no quería hacer, amenazando con lastimarla? (16\%); d) ¿Alguien ha actuado con celos extremos, como su dueño? (9\%); e ) ¿alguien ha usado un cuchillo, pistola u otra arma en contra suya? (2\%); f) ¿alguien le ha dado una cachetada, empujado, puesto contra el suelo, dado de puñetazos, pateado o tratado de estrangularla? (19\%); g) ¿alguien ha tenido sexo con usted cuando usted no quería? (7\%); h) ¿alguien la ha lastimado deliberadamente durante el sexo? (7\%); e i) ¿alguien la ha hecho sentir miedo de decir no al sexo? (7\%). Sólo el $14.7 \%$ de las mujeres que aceptaron violencia reportaron haber buscado alguna ayuda con la policía o personal de salud en forma previa. La mayoría de las mujeres reportaron que la persona que ejerció violencia fue su pareja (anterior/ actual); un porcentaje alto aceptó estar siendo víctima de abusos durante el embarazo actual (Tabla 2).

\section{Análisis adicional}

Al comparar las características socio-demográficas del grupo de mujeres que aceptó haber sido víctima de violencia versus no violencia se observó que vivir con pareja $\left(X^{2}=9.745, p=.002\right)$ fue la única variable que presentó significancia estadística.

Se observó la asociación de las variables continuas con violencia. La matriz de correlación muestra que violencia está asociada positivamente a actitud negativa durante el embarazo, tiempo de inicio del control prenatal, número de embarazos, y semanas en que demoró en sacar una cita para el control del embarazo; es decir a mayor experiencia con violencia la mujer tiene una actitud mas negativa hacia su embarazo, inicia más tarde su control prenatal, se demora en sacar cita para el CPN y el número de embarazos es mayor (Tabla 3).

\section{Discusión}

A través del análisis de factor se identificaron los componentes del CACM, logrando aglomerar los 9 reactivos en tres factores que correspondieron a violencia sexual, física y psicológica. Esto difiere con lo reportado por el autor original (Champion et al., 2004, Champion et al., 2005) que menciona que al realizar el análisis de factor con componentes principales y rotación Varimax produjo dos factores, uno con 6 reactivos relacionados con abuso sexual y otro con 4 reactivos relacionados con abuso físico y psicológico. Para interpretar esto se debe enfatizar en la clara asociación que se produjo en los datos obtenidos en esta población, identificándose visiblemente los reactivos que se agrupan más fuertemente entre ellos (Polit y Hungler, 2000). Esto nos indica que con los resultados de la aplicación del CACM no sólo identificamos mujeres con experiencia de violencia; sino también identificamos el tipo de violencia al cual ha estado expuesta la mujer.

El coeficiente de confiabilidad del CACM fue adecuado, indicando que mantiene congruencia interna; por lo que se ofrece como un instrumento válido para pesquisar violencia en la consulta clínica y también, para uso en investigación relacionada con violencia en contra de la mujer. La consistencia interna de CACM en este estudio se asemeja a lo reportado en otros estudios realizados en los EEUU con población afro-americana y Latina (Champion et al., 2004, Champion et al., 2005).

En este estudio, para facilitar el entendimiento y evitar barreras de alfabetización, el cuestionario fue leído en voz alta a la participante y se solicitó su respuesta. Siendo las respuestas de tipo dicotómicas, las participantes mencionaron que les fue fácil contestar. No obstante, este instrumento puede ser administrado a través de entrevista o de auto-respuesta. Algunos autores han sugerido que la auto-administración identifica mayor número de mujeres con violencia versus la entrevista individual (Kataoka, Yaku, Eto y Horiuch, 2010); sin embargo, es nuestra sugerencia que al momento de aplicar el cuestionario, los investigadores o personal de salud deben decidir la forma más apropiada considerando el componente cultural y/o nivel de alfabetización del grupo en que se quiere pesquisar violencia.

Se ha descrito que las mujeres que han sido víctimas de violencia tienen dificultad en reportarla ya sea por temor, vergüenza o porque piensan que es una situación que debe permanecer en los ámbitos del hogar; esta típica reacción impide que busquen ayuda (Flury et al., 2010; Humphreys et al., 2011; Keeling y Mason, 2011; Ravazzola, 1997). Un tercio de las participantes en este estudio reportaron haber sido víctima de violencia; sin embargo, un porcentaje mínimo refirió haber buscado ayuda previa; confirmándose la importancia de realizar pesquisa rutinaria como una forma de aumentar el diagnóstico y eventual intervención. Esto se asemeja a lo reportado por múltiples autores que indican que el embarazo representa una etapa propicia para realizar búsqueda rutinario de violencia (Bunn et al., 2009; Onifade et al., 2010; O'Reilly et al., 2010; Shay-Zapien y Bullock, 2010). Este estudio fue conducido de manera coherente con el cuidado rutinario que ofrecían clínicas de atención primaria en Monterrey, México. La aplicación del CACM en la primera visita durante este estudio sirvió para identificar 
muchas participantes que habían sido víctimas de violencia o que estaban viviendo en una relación de abuso. Enfermeras, parteras y personal de salud que están al cuidado de la salud de la mujer han mostrado ser expertos en comunicación, entendimiento y cuidados de salud; sin embargo, muchos de ellos carecen de conocimiento en cuanto al manejo de la violencia (Lazenbatt et al., 2009). Internacionalmente, los profesionales de salud requieren ser preparados para poder pesquisar, manejar y referir oportunamente los casos de violencia (Onifade et al, 2010; O'Reilly et al., 2010).

Otro antecedente que reporta el CACM es la persona que ejerce violencia en contra de la mujer. Más de la mitad del grupo de mujeres reportaron haber sido víctimas de violencia por parte de su pareja actual o previa. De hecho el $83 \%$ de la violencia física y el $93 \%$ de la violencia sexual fueron ejercidas por la pareja (anterior/actual).

Un importante hallazgo en este estudio fue que las mujeres que acusaban violencia presentaron una significativa asociación con actitud negativa hacia el embarazo y con CPN tardío; de hecho las semanas en que demoró en sacar su cita de CPN aumentaron a medida que aumenta su experiencia con violencia. Esto confirma hallazgos reportados por otros autores donde mujeres víctimas de violencia tienen más embarazos no deseados y por lo tanto retrasan el inicio del CPN (Miller et al, 2010; Moraes et al., 2010). También se encontró que mujeres que referían violencia vivían con su pareja y tenían mayor número de embarazos.

Se concluye que el CACM es un instrumento corto, de fácil manejo y que entrega suficiente información para determinar el tipo de violencia que está siendo víctima la mujer. Además, el CACM ayuda a identificar el perpetrador de violencia; si la mujer fue víctima de violencia en el pasado y si está aun viviendo en un ambiente de violencia. Este instrumento mostró consistencia interna y se ofrece como un instrumento válido para el uso en clínica e investigación. La pesquisa rutinaria de violencia en la primera visita aumentó considerablemente el reporte por parte de las mujeres embarazadas y sirvió para realizar intervenciones educativas de consejería y posteriormente referirlas a asistencia psicológica. La violencia se asoció significativamente a CPN tardío y embarazo no deseado. Se recomienda seguir poniendo a prueba el instrumento en otras poblaciones de América Latina como una forma de aumentar su validez en población que habla Español.

\section{Referencias}

Bunn, M. Y., Higa, N. A., Parker, W. J., y Kaneshiro, B. (2009). Domestic violence screening during pregnancy. Hawwaii Medical Journal, 68, 240-242.

Burns, N., y Grove, S. (2001). The practice of nursing research: Conduct, critique, and utilization. $4^{\mathrm{a}}$ ed. Philadelphia, Pennsylvania: Sanders Company.

Castro, R. (2004). Violencia contra mujeres embarazadas: tres estudios sociológicos. $1^{\text {a }}$ ed. Universidad Nacional Autónoma de México. Cuernavaca, Morelos.

Castro, R., Peek-Asa, C., y Ruiz, A. (2009). Violence against women in Mexico: A study of abuse before and during pregnancy. American Journal of Public Health,93, 1110-1116.

Champion, J. D. (1998). The context of sexually transmitted disease: life histories of women abuse. Issues of Mental Health Nursing, 19, 463-479.

Champion, J. D. (1999). Life histories of rural Mexican American adolescents experiencing abuse. Western Journal of Nursing Research, $21,688-717$.

Champion, J. D., Piper, J., Holden, A., Korte, J., y Shain, R. N. (2004). Abused women and risk for pelvic inflammatory disease. Western Journal of Nursing Research. 26, 176-191.

Champion, J. D., Piper, J. M., Holden, A. E. C., Shain, R. N., Perdue, S., y Korte, J. E. (2005). Relationship of abuse and pelvic inflammatory disease risk behavior in minority adolescents. Journal of American Nurse Practitioners, 17, 234-241.

Champion, J. D., Shain, R. N., Piper, J., y Perdue, S. T. (2001). Sexual abuse and sexual risk behaviors of minority women with sexually transmitted diseases. Western Journal of Nursing Research, 23, 241-254.

Chang, J., Berg, C. J., Saltzman, L. E., y Herndon, J. (2005). Homicide: A leading cause of injury deaths among pregnant and postpartum women in the United State 1991-1999. American Journal of Public Health, 95, 471-477.

Cheng, D., y Horon, I. L. (2010). Intimate partner homicide among pregnant and postpartum women. Obstetrics and Gynecology, 115, 1181-86.

Chu, S. Y., Goodwin, M. M., y D’Angelo, D. V. (2010). Physical violence against U.S. women around the time of pregnancy, 2004-2007. American $J$ of Preventive Medicine, 38, 317-322.

El-Mohandess, A. A., Kiely, M., Gantz, M. G., y El-Khorazaty, M. N. (2011). Very preterm birth is reduced in women receiving an integrated behavioral intervention: a randomized controlled trial. Maternaly Child Health Journal, 15, 19-28.

Flury, M., Nyberg, E., y Riecher-Rossler, A. (2010). Domestic violence against women: definitions, epidemiology, risk factors and consequences. Swiss Medical Weekly, 140:w13099.

Humphreys, J., Tsoh, J. Y., Kohn, M. A., y Gerbert, B. (2011). Increasing discussions of intimate partner violence in prenatal care using Video Doctor plus Provider Cueing: a randomized, controlled trial. Women's Health Issues, 21, 136-44.

Humphreys, J. (2011). Sexually transmitted infections, pregnancy, and intimate partner violence. Health Care for Women International, 32, 23-38.

Jacobson, N., y Gottman, J. (2001). Hombres que Agreden a sus Mujeres. $3^{\mathrm{a}}$ ed. Buenos Aires: Paidos.

Jasinski, J. L. (2004). Pregnancy and domestic violence: A review of the literature. Trauma, Violence, y Abuse, 5, 47-64.

Kataoka, Y., Yaju, Y., Eto, H., y Horiuchi, S. (2010). Self-administered questionnaire versus interview as a screening method for intimate partner violence in the prenatal setting in Japan: a randomized controlled trial. Pregnancy and Childbirth, 10, 84 . 
Keeling, J., y Mason, T. (2011). Postnatal disclosure of domestic violence: comparison with disclosure in the first trimester of pregnancy. Journal of Clinical Nursing, 20, 103-10.

Lazenbatt, A., Taylor, J., y Cree, L. (2009). A healthy setting framework: an evaluation and comparison of midwives' responses to addressing domestic violence. Midwifery, 25, 622-636.

Miller, E., Decker, M. R., McCauley, H. L., Tancredi, D. J., Levenson, R. R., Waldman, J. (2010). Pregnancy coercion, intimate partner violence and unintended pregnancy. Contraception, 81, 316-322.

Moraes, C. L., Arana, F. D., y Reichenheim, M. E. (2010). Physical intimate partner violence during gestation as a risk factor for low quality of pregnancy care. Revista de Saude Publica, 44, 667-676.

O’Reilly, R., Beale, B., y Gillies, D. (2010). Screening and intervention for domestic violence during pregnancy care: a systematic review. Trauma, Violence y Abuse, 11, 190-201.

Onifade, R., Von Rege, I., Mumford, S., y Bewley, S. (2010). Achieving continuous improvement in routine questioning about domestic violence with an embedded advocacy service. Journal of Obstetrics $y$ Gynaecology, 30, 550-552.

Organización Mundial de la Salud. (1996). Violence: A Public Health Priority. (WHO/EHA/SPI.POA.2). Geneva.

Pico-Alfonso, M. A., Garcia-Linares, M. I., Celda-Navarro, N., BlascoRos, C., Echeburua, E., y Martínez, M. (2006). The impact of physical, psychological, and sexual intimate male partner violence on women's mental health: depression symptoms, posttraumatic stress disorder, state anxiety, and suicide. Journal of Women's Health, 15, 599-610.

Polit, D. F., y Hungler, B. P. (2000). Investigación Cientifica en Ciencias de la Salud. México: McGraw-Hill Interamericana Editores, SA de CV.

Quelopana*, A. M., Champion, J. D., y Salazar, B. C. (2007). Factores psicosociales que influyen el inicio del control prenatal. Desarrollo Cientifico de Enfermería, 15, 102-106.
Quelopana*, A. M., Champion, J. D., y Salazar, B. C. (2009). Factors predicting the initiation of prenatal care in Mexican women. Midwifery, 25, 277-85.

Ravazzola, M. C. (1997). Historias Infames: El Maltrato en las Relaciones. Buenos Aires: Paidos.

Secretaria General de Salud de México. (2000). Norma Oficial de Criterios de Salud para el Cuidado Médico de la Violencia Familiar (NOM-190SSA1-1999). Ciudad de México, México.

Shah, P. S., y Shah, J. (2010). Maternal exposure to domestic violence and pregnancy and birth outcomes: a systematic review and meta-analyses. Journal of Women's Health, 19, 2017-2031.

Shay-Zapien, G., y Bullock, L. (2010). Impact of intimate partner violence on maternal child health. American Journal of Maternaly Child Nursing, 35, 206-212.

Stampfel, C. C., Chapman, D. A. y Álvarez, A. E. (2010). Intimate partner violence and posttraumatic stress disorder among high-risk women: does pregnancy matter? Violence Against Women, 16 , 426-443.

Taylor, R., y Nabors, E. L. (2009). Pink or blue... black and blue? Examining pregnancy as a predictor of intimate partner violence and femicide. Violence Against Women, 15, 1273-1293.

Thurman, A. R., Shain, R. N., Holden, A. E. C., Champion, J. D., Perdue, S. T., y Piper, J. M. (2008). Partner notification of sexually transmitted infections: a large cohort of Mexican American and African American women. Sexually Transmitted Diseases, 35, 136-140.

Valdez-Santiago, R., Arenas-Montreal, L. y Hernández-Tezoquipa, I. (2004). Experiencia de las parteras en la identificación de mujeres maltratadas durante el embarazo. Salud Pública de México, 46, 56-63.

Nota del autor: * Quelopana actualmente Linares 
